\title{
GENERIC PROPERTIES OF THE NICOLETTI AND FLOQUET BOUNDARY VALUE PROBLEMS
}

\author{
DARIUSZ BIELAWSKI
}

(Communicated by Hal L. Smith)

\begin{abstract}
The existence of solutions and the existence and uniqueness of solutions are considered as generic properties of an abstract boundary value problem. Applications to the Nicoletti and the Floquet boundary value problems are given.
\end{abstract}

This note is concerned with the system of ordinary differential equations with the Nicoletti or the Floquet boundary value condition. In [4] Lasota and Olech presented two results concerning the uniqueness of solutions and the existence of solutions of the Nicoletti boundary value problem. The sufficient conditions they gave are the best possible in a certain class of the right-hand sides of the system. Similar results for the Floquet boundary value problem were obtained by Kasprzyk and Myjak in [3].

In this note we widen the classes of the right-hand sides of these systems. The mentioned results are not true for all maps from the extended classes; however, we show that they are true for 'most'. More precisely, the subset of maps for which the existence of solutions or the existence and uniqueness of solutions fails to be true is of the first Baire category in an appropriate complete metric space. To do this we first prove two theorems which deal with generic properties of abstract boundary value problems.

In [1] there are presented results showing that under similar assumptions the set of solutions of the Nicoletti or the Floquet boundary value problem is an $R_{\delta}$-set.

Generic properties of functional and differential equations were studied by many authors. In Myjak's monograph [6] the reader can find several results and a list of references concerning this subject.

\section{Preliminaries}

In this note $E$ and $E_{1}$ denote two real Banach spaces (with norms $\|\cdot\|$ and $\|\cdot\|_{1}$ respectively). Let $X$ be a linear subspace of $E$, and let $L: X \rightarrow E_{1}$ be a linear (not necessarily continuous) map such that $\operatorname{Im} L=E_{1}$ and $\operatorname{Ker} L$ is a finite-dimensional space. Moreover, let $P: E \rightarrow \operatorname{Ker} L$ be a continuous projection onto $\operatorname{Ker} L$. For $\varphi: E \rightarrow E_{1}$ and $\xi \in \operatorname{Ker} L$ consider the boundary

Received by the editors June 29, 1992.

1991 Mathematics Subject Classification. Primary 47H15; Secondary 34B15.

(C)1994 American Mathematical Society 
value problem

$$
\left\{\begin{array}{l}
L(x)=\varphi(x) \\
P(x)=\xi
\end{array}\right.
$$

where $x \in X$. Many interesting results concerning the problem (1.1) (in a more general setting) the reader can find in [7]. In this note we study the existence of solutions and the existence and uniqueness of solutions as generic properties of the problem (1.1). As applications we obtain some results about the Nicoletti and Floquet boundary value problems.

We can associate with $L$ the right inverse $T: E_{1} \rightarrow E$ given as

$$
T(y)=x \quad \text { iff } \quad L(x)=y \text { and } P(x)=0 .
$$

This right-inverse can be defined by putting $T(y)=\tilde{x}-P(\tilde{x})$ for $y=L(\tilde{x})$. One can observe that $T$ is well defined and linear. Now we can write the problem (1.1) in an equivalent form, namely,

$$
x=\xi+T \circ \varphi(x), \quad x \in E .
$$

In what follows by $U$ we understand a neighbourhood of 0 in the space $E$ and by $n(E)$ the family of all nonempty subsets of $E$. We say that a multivalued map $H: U \rightarrow n(E)$ satisfies the Lasota-Opial conditions if:

(i) the graph $\{(x, y): y \in H(x)\}$ of $H$ is closed in $U \times E$,

(ii) $\overline{H(A)}$ is compact for every bounded $A \subset U$,

(iii) if $x \in U$ and $x \in H(x)$, then $x=0$.

In [5] Lasota and Opial proved the following

Theorem A. Suppose that $H: U \rightarrow n(E)$ satisfies the Lasota-Opial conditions. Then, for any continuous map $h: E \rightarrow E$ the condition

$$
h(x)-h(y) \in H(x-y) \text { for } x-y \in U
$$

implies that $I-h: E \rightarrow E$ is a homeomorphism $(I: E \rightarrow E$ denotes the identity map). Furthermore, for every $\varepsilon>0$ there exists $\delta>0$ such that for every $x \in E$

$$
(I-h)(B(x, \varepsilon)) \supset B(x-h(x), \delta)
$$

$(B(x, \varepsilon)$ denotes the open ball about $x$ of radius $\varepsilon)$.

In what follows we use the domain invariance theorem [2, p. 65]:

Theorem B. Let $\bar{B}(z, \varepsilon)$ be a closed ball in the space $E$, and let $g: \bar{B}(z, \varepsilon) \rightarrow E$ be a compact field (i.e., $I-g$ is a compact map). If $g$ is injective and

$$
\|x-y\|=\varepsilon \Rightarrow\|g(x)-g(y)\|>\delta,
$$

where $\delta$ is a positive constant, then

$$
g(B(z, \varepsilon)) \supset B(g(z), \delta) .
$$

By $(\mathscr{Z}, \rho)$ we mean a complete linear subspace of the space of all continuous $\varphi: E \rightarrow E_{1}$ such that $\varphi(A)$ is bounded for every bounded $A \subset E$, endowed with the metric

$$
\rho\left(\varphi_{1}, \varphi_{2}\right)=\sum_{n=1}^{\infty} 2^{-n} \frac{\sup \left\{\left\|\varphi_{1}(x)-\varphi_{2}(x)\right\|_{1}:\|x\| \leq n\right\}}{1+\sup \left\{\left\|\varphi_{1}(x)-\varphi_{2}(x)\right\|_{1}:\|x\| \leq n\right\}} .
$$




\section{General RESUlts}

Theorem 1. Let $H: U \rightarrow n(E)$ be a multivalued map with values starshaped with respect to 0 such that $H_{\lambda}: U \rightarrow n(E)$ given by $H_{\lambda}(x)=\lambda H(x)$ satisfies the Lasota-Opial conditions for every $\lambda \in[0,1)$. Moreover, let $(\mathscr{F}, \rho)$ be the subspace of the space $(\mathscr{Z}, \rho)$ defined by

$$
\mathscr{F}=\{\varphi \in \mathscr{Z}: x-y \in U \Rightarrow T(\varphi(x)-\varphi(y)) \in H(x-y)\} .
$$

Suppose that $T: E_{1} \rightarrow E$ is continuous. Then $\mathscr{F}^{*}$, the set of all $\varphi \in \mathscr{F}$ such that for every $\xi \in \operatorname{Ker} L$ the problem (1.1) has exactly one solution, is residual in the complete metric space $(\mathscr{F}, \rho)$.

Proof. Note that $H$ has all values closed in $E$. Since the space $(\mathscr{Z}, \rho)$ is complete and $\mathscr{F}$ is a closed subset of $\mathscr{Z}$, the space $(\mathscr{F}, \rho)$ is also complete. Define

(2.2) $\mathscr{M}=\{\varphi \in \mathscr{F}: \exists \lambda \in[0,1)(x-y \in U \Rightarrow T(\varphi(x)-\varphi(y)) \in \lambda H(x-y))\}$.

Observe that $\mathscr{M}$ is dense in $\mathscr{F}$. Indeed, if $\varphi \in \mathscr{F}$ then for $\varphi_{n}: E \rightarrow E_{1}$ given by $\varphi_{n}=n \varphi /(n+1)$ we have $\rho\left(\varphi_{n}, \varphi\right) \rightarrow 0$ as $n \rightarrow \infty$ and

$$
x-y \in U \Rightarrow T\left(\varphi_{n}(x)-\varphi_{n}(y)\right)=\frac{n}{n+1} T(\varphi(x)-\varphi(y)) \in \frac{n}{n+1} H(x-y),
$$

so from (2.2) we get $\varphi_{n} \in \mathscr{M}$ for $n \in \mathbf{N}$.

For every $\psi \in \mathscr{M}$ and $k \in \mathbf{N}$ we will assign a positive number $\varepsilon(\psi, k)$ in such a way that

$$
\bigcap_{k=0}^{\infty} \bigcup_{\psi \in \mathscr{M}} B(\psi, \varepsilon(\psi, k)) \subset \mathscr{F}^{*} .
$$

From this inclusion it follows that $\mathscr{F}^{*}$ contains a dense $G_{\delta}$-set, so $\mathscr{F}^{*}$ is residual in $\mathscr{F}$.

Suppose that $\psi \in \mathscr{M}$ and $k \in \mathbf{N}$. From (2.2) it follows that there exists $\lambda \in[0,1)$ such that

$$
x-y \in U \Rightarrow T \circ \psi(x)-T \circ \psi(y) \in H_{\lambda}(x-y) .
$$

Applying Theorem A to $T \circ \psi$ and $H_{\lambda}$ we get that $I-T \circ \psi: E \rightarrow E$ is a homeomorphism and the problem (1.1) has exactly one solution if we replace $\varphi$ by $\psi \in \mathscr{M}$. Furthermore, there exists $\delta(\psi, k) \in\left(0, \frac{1}{k+1}\right)$ satisfying

$$
\|x-y\| \geq \frac{1}{k+1} \Rightarrow\|x-T \circ \psi(x)-(y-T \circ \psi(y))\|>3 \delta(\psi, k) .
$$

From (1.3) and the continuity of linear $T$ it follows that one can find a positive $\varepsilon(\psi, k)$ such that for any $\varphi \in B(\psi, \varepsilon(\psi, k))$ we have

$$
\|x\| \leq 2 / \delta(\psi, k) \Rightarrow\|T(\varphi(x)-\psi(x))\|<\delta(\psi, k) .
$$

Now we are ready to show the inclusion (2.3). Suppose that $\varphi$ belongs to the left side of this inclusion. This means that there exists a sequence $\left\{\psi_{k}\right\}$ such that $\psi_{k} \in \mathscr{M}$ and $\rho\left(\psi_{k}, \varphi\right)<\varepsilon\left(\psi_{k}, k\right)$ for $k \in \mathbf{N}$. Define $g: E \rightarrow E$ by $g=I-T \circ \varphi$. From (2.4) and (2.5) it follows that if $x, y \in E$ satisfy $\|x\|,\|y\| \leq 2 / \delta\left(\psi_{k}, k\right)$ and $\|x-y\| \geq \frac{1}{k+1}$ then

$$
\begin{aligned}
\|g(x)-g(y)\| \geq & \left\|x-y-T\left(\psi_{k}(x)-\psi_{k}(y)\right)\right\| \\
& -\left(\left\|T\left(\psi_{k}(x)-\varphi(x)\right)\right\|+\left\|T\left(\psi_{k}(y)-\varphi(y)\right)\right\|\right) \\
& >\delta\left(\psi_{k}, k\right)>0 .
\end{aligned}
$$


Since one can choose $k \in \mathbf{N}$ arbitrarily and $2 / \delta\left(\psi_{k}, k\right)>2 k+2$, the map $g=I-T \circ \varphi$ is injective. Therefore, the problem (1.2) (and so the equivalent problem (1.1)) has at most one solution.

The map $H_{\lambda}=\lambda H$ satisfies the Lasota-Opial conditions for $\lambda \in[0,1)$, so $\overline{H(A)}$ is compact for every bounded $A \subset E$. Since $\varphi \in \mathscr{F}$, from (2.1) it follows that $T \circ \varphi$ is completely continuous.

Now consider the closed ball $\bar{B}\left(z, \frac{1}{k+1}\right) \subset E$, where $\|z\| \leq 1 / \delta\left(\psi_{k}, k\right)$. Since $g$ is injective and for $x, y \in \bar{B}\left(z, \frac{1}{k+1}\right)$ such that $\|x-y\|=\frac{1}{k+1}(2.6)$ holds, by Theorem B we have

$$
\|z\| \leq \frac{1}{\delta\left(\psi_{k}, k\right)} \Rightarrow g\left(B\left(z, \frac{1}{k+1}\right)\right) \supset B\left(g(z), \delta\left(\psi_{k}, k\right)\right) .
$$

From this and the inequality $\delta\left(\psi_{k}, k\right)<\frac{1}{k+1} \leq 1$ it follows that

$$
g\left(B\left(0,1 / \delta\left(\psi_{k}, k\right)\right)\right) \supset B(g(0), k) \text {. }
$$

Since one can choose $k \in \mathbf{N}$ arbitrarily, the map $g=I-T \circ \varphi$ is surjective. Therefore, the problem (1.2) (and so the equivalent problem (1.1)) has at least one solution, which finishes the proof.

Theorem 2. Let $H: E \rightarrow n(E)$ be a multivalued map with values starshaped with respect to 0 such that

$$
\lambda>0, \quad x \in E \Rightarrow H(\lambda x)=\lambda H(x)
$$

and $H_{\lambda}: E \rightarrow n(E)$ given by $H_{\lambda}=\lambda H$ satisfy the Lasota-Opial conditions for every $\lambda \in[0,1)$. Let $G: E \rightarrow n(E)$ be a multivalueld map with values closed and starshaped with respect to 0 and such that the set $\overline{\bigcup_{x \in E} G(x)}$ is compact. Moreover, let $(\mathscr{G}, \rho)$ be the subspace of the space $(\mathscr{Z}, \rho)$ defined by

$$
\mathscr{G}=\{\varphi \in \mathscr{Z}: x \in E \Rightarrow T \circ \varphi(x) \in H(x)+G(x)\} .
$$

Suppose that $T: E_{1} \rightarrow E$ is continuous. Then $\mathscr{G}^{*}$, the set of all $\varphi \in \mathscr{G}$ such that for every $\xi \in \operatorname{Ker} L$ the problem (1.1) has at least one solution, is residual in the complete space $(\mathscr{G}, \rho)$.

Proof. Since the space $(\mathscr{Z}, \rho)$ is complete and $\mathscr{G}$ is a closed subset of $\mathscr{Z}$, the space $(\mathscr{G}, \rho)$ is also complete. Define

$$
\mathscr{N}=\left\{\varphi \in \mathscr{G}: \exists \lambda \in[0,1)\left(x \in E \Rightarrow T \circ \varphi(x) \in H_{\lambda}(x)+G(x)\right)\right\} .
$$

Note that $\mathscr{N}$ is dense in $\mathscr{G}$. Indeed, if $\varphi \in \mathscr{G}$ then for $\varphi_{n}: E \rightarrow E_{1}$ given by $\varphi_{n}=n \varphi /(n+1)$ we have $\rho\left(\varphi_{n}, \varphi\right) \rightarrow 0$ as $n \rightarrow \infty$ and

$$
x \in E \Rightarrow T \circ \varphi_{n}(x)=\frac{n}{n+1} T(\varphi(x)) \in \frac{n}{n+1} H(x)+\frac{n}{n+1} G(x),
$$

so $\varphi_{n} \in \mathscr{N}$.

Suppose that $\psi \in \mathscr{N}$. Then there exists $\lambda \in[0,1)$ such that

$$
x \in E \Rightarrow T \circ \psi(x) \in H_{\lambda}(x)+G(x) .
$$

We claim that

$$
\begin{aligned}
& \forall K \geq 0 \exists M>0 \forall x, y \in E \forall \mu \geq 1 \\
& \quad(\|x\|=M \wedge\|y\| \leq K \Rightarrow \mu x \neq T \circ \psi(x)+y) .
\end{aligned}
$$


To prove this, suppose the contrary. This means that there exist sequences $\left\{x_{m}\right\} \subset E,\left\{y_{m}\right\} \subset E,\left\{\mu_{m}\right\} \subset[1, \infty)$ such that $\left\|x_{m}\right\| \rightarrow \infty$ as $m \rightarrow \infty$, $\left\|y_{m}\right\| \leq K$ for $m \in \mathbf{N}$, and

$$
\mu_{m} x_{m}=T \circ \psi\left(x_{m}\right)+y_{m}, \quad m \in \mathbf{N} .
$$

Using assumptions imposed on $H_{\lambda}$ and $G$ and putting $w_{m}=x_{m} /\left\|x_{m}\right\|$ we obtain

$$
w_{m}-\frac{y_{m}}{\mu_{m}\left\|x_{m}\right\|} \in H_{\lambda}\left(w_{m}\right)+\frac{G\left(x_{m}\right)}{\left\|x_{m}\right\|}, \quad m \in \mathbf{N} .
$$

Since $y_{m} /\left(\mu_{m}\left\|x_{m}\right\|\right) \rightarrow 0, \sup \left\{\|z\|: z \in G\left(x_{m}\right) /\left\|x_{m}\right\|\right\} \rightarrow 0$ as $m \rightarrow \infty$, and $\overline{H_{\lambda}(\bar{B}(0,1))}$ is compact, we have passing if necessary to a subsequence that $w_{m} \rightarrow w$ as $m \rightarrow \infty$. The graph of $H_{\lambda}$ is closed, so $w \in H_{\lambda}(w)$, which implies that $w=0$. But $\left\|w_{m}\right\|=1$ for $m \in \mathbf{N}$ and $\|w\|=1$, which leads to a contradiction.

From the condition (2.9) it follows that $I-T \circ \psi: E \rightarrow E$ is a surjective map. In fact, if $\|y\|=K$, then define a continuous retraction $\tau: E \rightarrow \bar{B}(0, M)$ by

$$
\tau(x)= \begin{cases}x, & \|x\| \leq M, \\ M x /\|x\|, & \|x\|>M,\end{cases}
$$

where $M$ is a constant satisfying the condition (2.9). Define also a compact map $h: E \rightarrow E$ by

$$
h(x)=T \circ \psi \circ \tau(x)+y .
$$

This map must have a fixed point $x_{0}$, but, since (2.9) holds, this point cannot lie outside the open ball $B(0, M)$. Thus, $\tau\left(x_{0}\right)=x_{0}$ and $x_{0}$ is also a fixed point of the map $T \circ \psi+y$, so $(I-T \circ \psi)\left(x_{0}\right)=y$.

For every $\psi \in \mathscr{N}$ and every $k \in \mathbf{N}$ we will assign a positive number $\eta(\psi, k)$ in such a way that

$$
\bigcap_{k=0}^{\infty} \bigcup_{\psi \in \mathscr{N}} B(\psi, \eta(\psi, k)) \subset \mathscr{G}^{*} .
$$

Suppose that $\psi \in \mathscr{N}$ and $k \in \mathbf{N}$. Let $M(\psi, k)$ be such a positive constant that for all $\mu \geq 1,\|x\|=M(\psi, k)$, and $\|y\| \leq k$ we have $\mu x \neq T \circ \psi(x)+y$ (the existence of $M(\psi, k)$ is guaranteed by (2.9)). Since $T \circ \psi$ is completely continuous and $\operatorname{Ker} L$ is finite dimensional, one can find a positive $\delta(\psi, k)$ such that

$$
\begin{gathered}
\mu \geq 1, \quad \xi \in \operatorname{Ker} L,\|\xi\| \leq k,\|x\|=M(\psi, k) \\
\Rightarrow\|\mu x-T \circ \psi(x)-\xi\|>\delta(\psi, k) .
\end{gathered}
$$

From (1.3) and the continuity of linear $T$ it follows that one can find a positive $\eta(\psi, k)$ such that for any $\varphi \in B(\psi, \eta(\psi, k))$ we have

$$
\|x\|=M(\psi, k) \Rightarrow\|T(\varphi(x)-\psi(x))\|<\frac{1}{2} \delta(\psi, k) .
$$

Finally we show the inclusion (2.10). Suppose that $\varphi$ belongs to the left side of (2.10). This means that there exists a sequence $\left\{\psi_{k}\right\}$ such that $\psi_{k} \in \mathscr{N}$ and $\rho\left(\psi_{k}, \varphi\right)<\eta\left(\psi_{k}, k\right)$ for $k \in \mathbf{N}$. From (2.11) and (2.12) it follows that for $\mu \geq 1,\|x\|=M\left(\psi_{k}, k\right), \xi \in \operatorname{Ker} L$, and $\|\xi\| \leq k$ we have

$$
\|\mu x-T \circ \varphi(x)-\xi\|>\frac{1}{2} \delta\left(\psi_{k}, k\right) .
$$


Since we can choose $k \in \mathbf{N}$ arbitrarily, we have

$$
\begin{aligned}
& \forall K \geq 0 \exists M>0 \forall x \in E \forall \xi \in \operatorname{Ker} L \forall \mu \geq 1 \\
& \quad(\|x\|=M \wedge\|\xi\| \leq K \Rightarrow \mu x \neq T \circ \varphi(x)+\xi) .
\end{aligned}
$$

Using this condition one can show, similarly as for $\psi \in \mathscr{N}$, that $(I-T \circ \varphi)(E) \supset$ $\operatorname{Ker} L$. Therefore, the problem (1.2) (and so the equivalent problem (1.1)) has at least one solution, which proves the inclusion (2.10). From this inclusion it follows that $\mathscr{G}^{*}$ contains a dense $G_{\delta}$-set, so $\mathscr{G}^{*}$ is residual in $\mathscr{G}$, which finishes the proof.

\section{The NicoletTI PROBLEM}

We say that $f:[a, b] \times \mathbf{R}^{d} \rightarrow \mathbf{R}^{d}$ is a Caratheodory map if $f(\cdot, x):[a, b] \rightarrow$ $\mathbf{R}^{d}$ is measurable for every $x \in \mathbf{R}^{d}$ and $f(t, \cdot): \mathbf{R}^{d} \rightarrow \mathbf{R}^{d}$ is continuous for every $t \in[a, b]$. Consider the Nicoletti problem

$$
\left\{\begin{array}{l}
x^{\prime}(t)=f(t, x(t)) \text { a.e. on }[a, b], \\
N(x)=r
\end{array}\right.
$$

where $f:[a, b] \times \mathbf{R}^{d} \rightarrow \mathbf{R}^{d}$ is a Caratheodory map, $r \in \mathbf{R}^{d}$, and $N: C\left([a, b], \mathbf{R}^{d}\right)$ $\rightarrow \mathbf{R}^{d}$ is given by $N(x)=\left(x_{1}\left(t_{1}\right), x_{2}\left(t_{2}\right), \ldots, x_{d}\left(t_{d}\right)\right)$, where $t_{1}, t_{2}, \ldots, t_{d} \in$ $[a, b]$. By a solution of $(3.1)$ we mean any absolutely continuous map $x:[a, b]$ $\rightarrow \mathbf{R}^{d}$ satisfying (3.1).

In what follows by $E$ we denote the space $C\left([a, b], \mathbf{R}^{d}\right)$ of all continuous maps from $[a, b]$ to $\mathbf{R}^{d}$ with the norm $\|x\|=\sup \{|x(t)|: t \in[a, b]\}$, where $|\cdot|$ denotes the Euclidean norm in $\mathbf{R}^{d}$. By $X$ we mean the linear subspace of $E$ of all absolutely continuous $x:[a, b] \rightarrow \mathbf{R}^{d}$. By $E_{1}$ we denote the space $L^{1}\left([a, b], \mathbf{R}^{d}\right)$ of all Lebesgue integrable maps from $[a, b]$ to $\mathbf{R}^{d}$ with the norm $\|x\|_{1}=\int_{a}^{b}|x(t)| d t$.

Now consider the space $(\widetilde{\mathcal{Z}}, \tilde{\rho})$ of all Carathéodory maps $f:[a, b] \times \mathbf{R}^{d} \rightarrow$ $\mathbf{R}^{d}$ such that

$$
\int_{a}^{b} \sup \{|f(t, u)|:|u| \leq n\} d t<\infty \text { for } n \in \mathbf{N}
$$

endowed with the metric $\tilde{\rho}$ given by

$$
\tilde{\rho}\left(f_{1}, f_{2}\right)=\sum_{n=1}^{\infty} 2^{-n} \frac{\int_{a}^{b} \sup \left\{\left|f_{1}(t, u)-f_{2}(t, u)\right|:|u| \leq n\right\} d t}{1+\int_{a}^{b} \sup \left\{\left|f_{1}(t, u)-f_{2}(t, u)\right|:|u| \leq n\right\} d t} .
$$

We identify maps which differ only for $t$ belonging to a set of measure zero. One can observe that this space is complete.

Let $(\mathscr{Z}, \rho)$ be the space of all continuous maps $\varphi: E \rightarrow E_{1}$ such that there exists a Carathéodory map $f \in \widetilde{\mathscr{Z}}$ satisfying

$$
x \in E \Rightarrow \varphi(x)(t)=f(t, x(t)) \text { a.e. on }[a, b]
$$

with the metric $\rho$ given by (1.3).

Lemma. Spaces $(\mathscr{Z}, \rho)$ and $(\widetilde{Z}, \tilde{\rho})$ are isometric.

Proof. We will show that $J: \widetilde{\mathscr{Z}} \rightarrow \mathscr{Z}$ defined by associating with $f \in \widetilde{\mathscr{Z}}$ the map $\varphi \in \mathscr{Z}$ by (3.2) is an isometry. It suffices to prove that for every $f \in \widetilde{Z}$ 
and $n \in \mathbf{N}$ we have

$$
\int_{a}^{b} \sup \{|f(t, u)|:|u| \leq n\} d t=\sup \left\{\int_{a}^{b}|f(t, x(t))| d t:\|x\| \leq n\right\} .
$$

Let $\left\{u_{m}\right\}$ be a sequence which is dense in $\bar{B}(0, n) \subset \mathbf{R}^{d}$. Notice that

$$
\lim _{m \rightarrow \infty} \int_{a}^{b} \max \left\{|f(t, u)|: u=u_{1}, \ldots, u_{m}\right\} d t=\int_{a}^{b} \sup \{|f(t, u)|:|u| \leq n\} d t .
$$

For $i=1, \ldots, m$ define sets $A_{m, i}$ in such a way that $t \in[a, b]$ belongs to $A_{m, i}$ iff

$$
\left|f\left(t, u_{i}\right)\right|=\max \left\{|f(t, u)|: u=u_{1}, \ldots, u_{m}\right\}
$$

and

$$
j<i \Rightarrow\left|f\left(t, u_{i}\right)\right|>\left|f\left(t, u_{j}\right)\right| \text {. }
$$

One can observe that for a fixed $m$ sets $A_{m, i}$ are measurable, disjoint, and $\bigcup_{i=1}^{m} A_{m, i}=[a, b]$. Define an integrable map $\tilde{x}_{m}:[a, b] \rightarrow \mathbf{R}^{d}$ by

$$
\tilde{x}_{m}(t)=u_{i} \Leftrightarrow t \in A_{m, i} \text {. }
$$

Now from Luzin's theorem we obtain that for every $m \in \mathbf{N}$ there exists a continuous map $x_{m}:[a, b] \rightarrow \mathbf{R}^{d}$ such that $\left.x_{m}\right|_{D_{m}}=\left.\tilde{x}_{m}\right|_{D_{m}}$, where $D_{m}$ is closed in $[a, b]$ and $\lim _{m \rightarrow \infty} \mu\left(D_{m}\right)=b-a$. One can check that

$$
\lim _{m \rightarrow \infty} \int_{a}^{b}\left|f\left(t, x_{m}(t)\right)\right| d t=\int_{a}^{b} \sup \{|f(t, u)|:|u| \leq n\} d t
$$

Therefore, we have

$$
\int_{a}^{b} \sup \{|f(t, u)|:|u| \leq n\} d t \leq \sup \left\{\int_{a}^{b}|f(t, x(t))| d t:\|x\| \leq n\right\} .
$$

This completes the proof since the inverse inequality is obvious.

In [4] Lasota and Olech proved the following

Theorem C. Suppose that $p, q:[a, b] \rightarrow[0, \infty)$ are Lebesgue integrable and $p$ satisfies the inequality

$$
\int_{a}^{b} p(t) d t<\frac{\pi}{2}
$$

Then:

(i) the map $x \equiv 0$ is the only one absolutely continuous of $[a, b]$ into $\mathbf{R}^{d}$ which satisfies the conditions

$$
\begin{gathered}
N(x)=0, \\
\left|x^{\prime}(t)\right| \leq p(t)|x(t)| \text { a.e. on }[a, b] ;
\end{gathered}
$$

(ii) if a Carathéodory map $f:[a, b] \times \mathbf{R}^{d} \rightarrow \mathbf{R}^{d}$ satisfies the inequality

$$
|f(t, u)-f(t, v)| \leq p(t)|u-v| \text { for } u, v \in \mathbf{R}^{d}, t \in[a, b],
$$

and $f(\cdot, 0)$ is integrable, then the problem (3.1) has exactly one solution; 
(iii) if a Carathéodory map $f:[a, b] \times \mathbf{R}^{d} \rightarrow \mathbf{R}^{d}$ satisfies the inequality

$$
|f(t, u)| \leq p(t)|u|+q(t) \quad \text { for } u \in \mathbf{R}^{d}, t \in[a, b],
$$

then the problem (3.1) has at least one solution.

An example in [4] shows that ' $<$ ' in (3.3) cannot be replaced by ' $\leq$ '. However, such a situation is to be considered quite exceptional in view of the following results.

Theorem 3. Assume that $p:[a, b] \rightarrow[0, \infty)$ is an integrable map satisfying the inequality

$$
\int_{a}^{b} p(t) d t \leq \frac{\pi}{2}
$$

Let $\mathscr{F}_{1}$ denote the subset of $\widetilde{Z}$ of all $f$ satisfying

$$
|f(t, u)-f(t, v)| \leq p(t)|u-v| \text { for } u, v \in \mathbf{R}^{d}, t \in[a, b] .
$$

Then $\mathscr{F}_{1}^{*}$, the set of all $f \in \mathscr{F}_{1}$ such that for every $r \in \mathbf{R}^{d}$ the problem (3.1) has exactly one solution, is residual in the complete space $\left(\mathscr{F}_{1}, \tilde{\rho}\right)$.

Proof. If we put $\varphi=J(f), L(x)(t)=x^{\prime}(t), \xi(t)=r$, and $P(x)(t)=N(x)$ for $t \in[a, b]$, then the problem (3.1) becomes equivalent to the problem (1.1). The continuous right inverse $T: E_{1} \rightarrow E$ of $L$ is given by

$$
T(y)(t)_{i}=\int_{t_{i}}^{t} y_{i}(s) d s, \quad i=1,2, \ldots, d .
$$

Define the multivalued map $H: E \rightarrow n(E)$ by

$$
\begin{aligned}
H(x)=\{z \in E: & N(z)=0, a \leq s \leq t \leq b \\
& \left.\Rightarrow|z(t)-z(s)| \leq \int_{s}^{t} p(u)|x(u)| d u\right\} .
\end{aligned}
$$

Note that $H$ has values starshaped with respect to 0 . Furthermore, $H_{\lambda}=\lambda H$ satisfies the Lasota-Opial conditions for every $\lambda \in[0,1)$. In fact, if $x \in H_{\lambda}(x)$ then from (3.5) it follows that $N(x)=0$ and

$$
\left|x^{\prime}(t)\right| \leq \lambda p(t)|x(t)| \text { a.e. on }[a, b],
$$

so by Theorem $\mathrm{C}$ we have that $x=0$. Two remaining conditions also follows from the definitions of $H$ and $H_{\lambda}$.

Now using Theorem 1 we have that $\mathscr{F}^{*}$, the set of all $\varphi \in \mathscr{F}$ such that for every $\xi \in \operatorname{Ker} L$ the problem (1.1) has exactly one solution, is residual in the complete space $(\mathscr{F}, \rho)$, where $\mathscr{F}$ is given by $(2.1)$.

Observe that $J\left(\mathscr{F}_{1}\right)=\mathscr{F}$. In fact, it is obvious that $J\left(\mathscr{F}_{1}\right) \subset \mathscr{F}$. Now suppose that $J(f) \in \mathscr{F}$, where $f \in \widetilde{\mathscr{Z}}$. This says that

$$
\left|\int_{s}^{t}(f(u, x(u))-f(u, y(u))) d u\right| \leq \int_{s}^{t} p(u)|x(u)-y(u)| d u
$$

for all $a \leq s \leq t \leq b$ and $x, y \in E$. Choose a sequence $\left\{\left(v_{m}, w_{m}\right)\right\}$ dense in $\mathbf{R}^{d} \times \mathbf{R}^{d}$. Substituting $v_{m}$ and $w_{m}$ for $x(u)$ and $y(u)$ in (3.6), dividing this inequality by $|t-s|$, and passing $t \rightarrow s$ yields that the set of $s$ satisfying

$$
\left|f\left(s, v_{m}\right)-f\left(s, w_{m}\right)\right| \leq p(s)\left|v_{m}-w_{m}\right|
$$


for a fixed $m \in \mathbf{N}$ has measure $b-a$. Therefore, the measure of the set of $s$ satisfying (3.7) for all $m \in \mathbf{N}$ has also measure $b-a$. This means that $f \in \mathscr{F}_{1}$ because $f(s, \cdot)$ is continuous for every $s \in[a, b]$. Since $J\left(\mathscr{F}_{1}\right)=\mathscr{F}$ and $J$ is an isometry, we find that $\mathscr{F}_{1}^{*}=J^{-1}\left(\mathscr{F}^{*}\right)$ is residual in $\mathscr{F}_{1}$, which completes the proof.

Theorem 4. Assume that $p, q:[a, b] \rightarrow[0, \infty)$ are integrable maps and $p$ satisfies the inequality (3.4). Let $\mathscr{G}_{1}$ denote the subset of $\widetilde{\mathscr{Z}}$ of all $f$ satisfying

$$
|f(t, u)| \leq p(t)|u|+q(t) \quad \text { for } u \in \mathbf{R}^{d}, t \in[a, b] .
$$

Then $\mathscr{G}_{1}^{*}$, the set of all $f \in \mathscr{G}_{1}$ such that for every $r \in \mathbf{R}^{d}$ the problem (3.1) has at least one solution, is residual in the complete space $\left(\mathscr{G}_{1}, \tilde{\rho}\right)$.

Proof. As before, by putting $\varphi=J(f), L(x)(t)=x^{\prime}(t), \xi(t)=r$, and $P(x)(t)=N(x)$ for $t \in[a, b]$ we consider problem (1.1) instead of the problem (3.1). We define the maps $T: E_{1} \rightarrow E$ and $H: E \rightarrow n(E)$ as in the proof of Theorem 3. The multivalued map $G: E \rightarrow n(E)$ we define by

$$
G(x)=\left\{z \in E: N(z)=0, a \leq s \leq t \leq b \Rightarrow|z(t)-z(s)| \leq \int_{s}^{t} q(u) d u\right\} .
$$

Note that $G$ has closed values starshaped with respect to 0 and compact image. Furthermore, $H$ satisfies (2.7). Now using Theorem 2 we have that $\mathscr{G}^{*}$, the set of all $\varphi \in \mathscr{G}$ such that for every $r \in \operatorname{Ker} L$ the problem (1.1) has at least one solution, is residual in the space $(\mathscr{G}, \rho)$, where $\mathscr{G}$ is given by (2.8). Note that $J\left(\mathscr{G}_{1}\right)=\mathscr{G}$. In fact, if $f \in \mathscr{G}_{1}$, then define $\varphi_{1}, \varphi_{2}: E \rightarrow E_{1}$ by

$$
\varphi_{1}(x)(t)=\frac{p(t)|x(t)| f(t, x(t))}{p(t)|x(t)|+q(t)}, \quad \varphi_{2}(x)(t)=\frac{q(t) f(t, x(t))}{p(t)|x(t)|+q(t)}
$$

(if the denominator is 0 , we set $\varphi_{1}(x)(t)=0$ and $\varphi_{2}(x)(t)=f(t, x(t))$ ). One can see that $J(f)=\varphi_{1}+\varphi_{2}$ and $T \circ \varphi_{1}(x) \in H(x), T \circ \varphi_{2}(x) \in G(x)$ for $x \in E$, so $J\left(\mathscr{G}_{1}\right) \subset \mathscr{G}$. The inverse inclusion can be proved similarly as the inclusion $J\left(\mathscr{F}_{1}\right) \supset \mathscr{F}$ in the proof of Theorem 3. Since $J$ is an isometry, the set $\mathscr{G}_{1}^{*}=J^{-1}\left(\mathscr{G}^{*}\right)$ is residual in $\mathscr{G}_{1}$, which finishes the proof.

\section{The Floquet PRoblem}

Consider the Floquet problem

$$
\left\{\begin{array}{l}
x^{\prime}(t)=f(t, x(t)) \text { a.e. on }[a, b], \\
F(x)=r
\end{array}\right.
$$

where $f:[a, b] \times \mathbf{R}^{d} \rightarrow \mathbf{R}^{d}$ is a Carathéodory map, $r \in \mathbf{R}^{d}$, and $F$ : $C\left([a, b], \mathbf{R}^{d}\right) \rightarrow \mathbf{R}^{d}$ is given by $F(x)=x(a)+\zeta x(b)$, where $\zeta$ is a positive constant. By a solution of (4.1) we mean any absolutely continuous map $x:[a, b] \rightarrow \mathbf{R}^{d}$ satisfying (4.1).

Spaces $E, X, E_{1},(\mathscr{Z}, \rho),(\widetilde{Z}, \tilde{\rho})$ and the isometry $J: \widetilde{Z} \rightarrow \mathscr{Z}$ we define in the same way as in the $\S 3$. In [3] Kasprzyk and Myjak proved the following.

Theorem D. Suppose that $p, q:[a, b] \rightarrow[0, \infty)$ are Lebesgue integrable and $p$ satisfies the inequality

$$
\int_{a}^{b} p(t) d t<\sqrt{\pi^{2}+\ln ^{2} \zeta}
$$


Then

(i) the map $x \equiv 0$ is the only one absolutely continuous of $[a, b]$ into $\mathbf{R}^{d}$ which satisfies the conditions

$$
\begin{gathered}
F(x)=0, \\
\left|x^{\prime}(t)\right| \leq p(t)|x(t)| \text { a.e. on }[a, b]
\end{gathered}
$$

(ii) if a Carathéodory map $f:[a, b] \times \mathbf{R}^{d} \rightarrow \mathbf{R}^{d}$ satisfies the inequality

$$
|f(t, u)-f(t, v)| \leq p(t)|u-v| \text { for } u, v \in \mathbf{R}^{d}, t \in[a, b],
$$

and $f(\cdot, 0)$ is integrable, then the problem (4.1) has exactly one solution;

(iii) if a Carathéodory map $f:[a, b] \times \mathbf{R}^{d} \rightarrow \mathbf{R}^{d}$ satisfies the inequality

$$
|f(t, u)| \leq p(t)|u|+q(t) \quad \text { for } u \in \mathbf{R}^{d}, t \in[a, b],
$$

then the problem (4.1) has at least one solution.

An example in [3] shows that ' $<$ ' in (4.2) cannot be replaced by ' $\leq$ '.

Theorem 5. Assume that $p:[a, b] \rightarrow[0, \infty)$ is an integrable map satisfying the inequality

$$
\int_{a}^{b} p(t) d t \leq \sqrt{\pi^{2}+\ln ^{2} \zeta}
$$

Let $\mathscr{F}_{2}$ denote the subset of $\widetilde{Z}$ of all $f$ satisfying

$$
|f(t, u)-f(t, v)| \leq p(t)|u-v| \text { for } u, v \in \mathbf{R}^{d}, t \in[a, b] .
$$

Then $\mathscr{F}_{2}^{*}$, the set of all $f \in \mathscr{F}_{2}$ such that for every $r \in \mathbf{R}^{d}$ the problem (4.1) has exactly one solution, is residual in the complete space $\left(\mathscr{F}_{2}, \tilde{\rho}\right)$.

Proof. This theorem can be proved similarly to Theorem 3; the continuous projection $P: E \rightarrow \operatorname{Ker} L$ is given by $P(x)(t)=\frac{1}{1+\zeta} F(x)$ and the continuous right inverse $T: E_{1} \rightarrow E$ of $L$ by

$$
T(y)(t)=\frac{1}{1+\zeta} \int_{a}^{t} y(s) d s-\frac{\zeta}{1+\zeta} \int_{t}^{b} y(s) d s
$$

Theorem 6. Assume that $p, q:[a, b] \rightarrow[0, \infty)$ are integrable maps and $p$ satisfies the inequality (4.3). Let $\mathscr{G}_{2}$ denote the subset of $\widetilde{Z}$ of all $f$ satisfying

$$
|f(t, u)| \leq p(t)|u|+q(t) \quad \text { for } u \in \mathbf{R}^{d}, t \in[a, b] .
$$

Then $\mathscr{G}_{2}^{*}$, the set of all $f \in \mathscr{G}_{2}$ such that for every $r \in \mathbf{R}^{d}$ the problem (4.1) has at least one solution, is residual in the complete space $\left(\mathscr{G}_{2}, \tilde{\rho}\right)$.

The proof is similar to the proof of Theorem 4.

\section{ACKNOWLEDGMENT}

The author is grateful to Professor Tadeusz Pruszko for inspiration and help during preparation of this article. 


\section{REFERENCES}

1. D. Bielawski and T. Pruszko, On the structure of the set of solutions of a functional equation with applications to boundary value problems, Ann. Polon. Math. 53.3 (1991), 201-209.

2. J. Dugundji and A. Granas, Fixed point theory, PWN, Warsaw, 1982.

3. S. Kasprzyk and J. Myjak, On the existence of solutions of the Floquet problem for ordinary differential equations, Zeszyty Nauk. Uniw. Jagielloń. Prace Mat. 13 (1969), 35-39.

4. A. Lasota and C. Olech, An optimal solution of Nicoletti's boundary value problem, Ann. Polon. Math. 18 (1966), 131-139.

5. A. Lasota and Z. Opial, On the existence and uniqueness of solutions of nonlinear functional equations, Bull. Acad. Polon. Sci. Sér. Sci. Math. Astronom. Phys. 15 (1967), 797-800.

6. J. Myjak, Orlicz type category theorems for functional and differential equations, Dissertationes Math. (Rozprawy Mat.) 206 (1983).

7. T. Pruszko, Some applications of the topological degree theory to multi-valued boundary value problems, Dissertationes Math. (Rozprawy Mat.) 229 (1984).

Institute of Mathematics, University of GdaŃsk, Ul. Wita Stwosza 57, 80-952 GdaŃsK, POLAND

E-mail address: matdb@halina.univ.gda.pl 